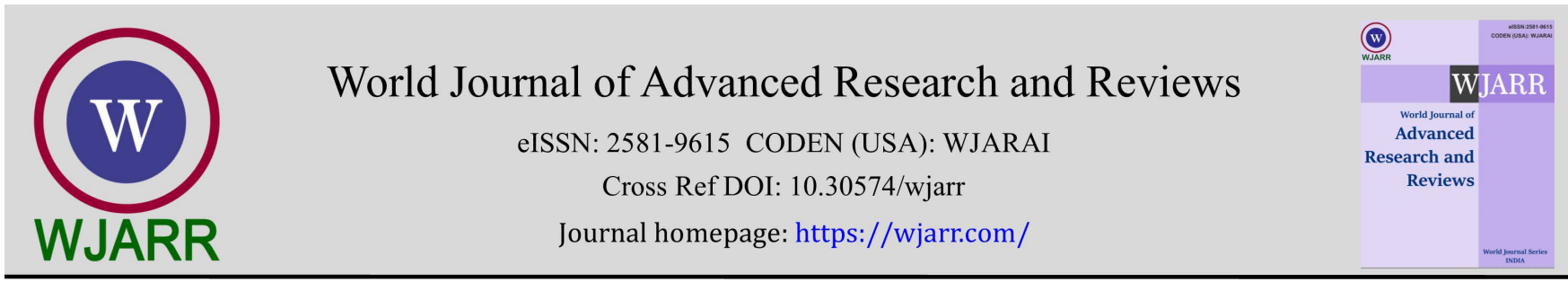

(RESEARCH ARTiClE)

Check for updates

\title{
The fading affect bias (FAB) is strongest for Jews and Buddhists and weakest for participants without religious affiliations: An exploratory analysis
}

Jeffrey A. Gibbons *, Sherman A. Lee, Luke P. Fernandez, Emma D. Friedmann, Kaylee D. Harris, Hannah E. Brown, and Rachel D. Prohaska

Christopher Newport University Psychology Department Avenue of the Arts Newport News, VA 23606 United States of America.

World Journal of Advanced Research and Reviews, 2021, 09(03), 350-362

Publication history: Received on 14 February 2021; revised on 18 March 2021; accepted on 20 March 2021

Article DOI: https://doi.org/10.30574/wjarr.2021.9.3.0109

\begin{abstract}
The fading affect bias (FAB) is a robust phenomenon where unpleasant affect fades faster than pleasant affect. The FAB is believed to be coping mechanism designed to make life appear pleasant in the face of hardships and adversities. The FAB persists across several cultures and many event types (e.g., alcohol, religious, and death), even though low FAB has been demonstrated for social media events, videogame events, and events labeled as religious, but not spiritual. Although religion is also believed to make life more satisfying by providing existential meaning and social connectedness for their followers, research to date, has not examined religious differences in the FAB. Therefore, we examined the FAB using 2 measures of fading affect across participants' self-reported religious affiliations and we found robust FAB effects for all categories except for an extremely small sample of Islamic followers. The FAB effects were strongest for Jewish and Buddhist affiliations and they were weakest for participants who did not report a well-known religious affiliation. The findings extend the literature on the FAB to religious belief systems. Future research should replicate the current study, examine the FAB for larger samples of Muslims, Buddhists, Hindus, and Jews, and test explanations for differential FAB effects across religious affiliations.
\end{abstract}

Keywords: Fading affect; Fading affect bias; General healthy coping; Religious affiliations

\section{Introduction}

Research on autobiographical memory shows that emotions tied to events differentially wane over time, such that unpleasant affect fades faster than pleasant affect [1]. This phenomenon is referred to as the fading affect bias [2]. The FAB is positively related to healthy outcomes, such as self-esteem [3] and positive religious coping [4], and it is negatively related to unhealthy outcomes, such as dysphoria [5] and trait anxiety [6]. In addition, the FAB persists across several different cultures [7]. Ritchie et al. [7] contended that the FAB was a healthy mechanism in memory that enhances pleasantness, which echoed similar arguments made in the literature [2, 8]. Although the FAB persisted across various events, the effect was small for social media events [3], videogame events [9], and religious events that were not spiritual [4]. The importance of religiousness for the FAB indicated that the phenomenon may vary across religious categories. Therefore, the goal of the current study was to examine the FAB across participants' self-reported religious affiliations.

The seminal work on fading emotions in autobiographical memory was done long ago by researchers who found that participants recorded and recalled a greater number of pleasant events than unpleasant events $[10,11,12,13]$ and that unpleasant affect diminished more over time than pleasant affect $[14,15]$. More recent research found similar patterns of differential fading affect [1], referred to as the fading affect bias [5], which began around 12 to 24 hours after an event, it persisted for 3 months [16], and it increased after that time [1]. Based on the persistent FAB effects across cultures 
[7], Ritchie et al. [7] argued that it may make life pleasant by reducing unpleasant affect and maintaining pleasant affect; these arguments resonated with the contentions already expressed in the FAB literature [17]. These arguments also fit well with emotion regulation theories focused on self-enhancement [18], which may occur through mobilized resources and minimized unpleasantness [19].

The literature generally supports the notion that the FAB is a healthy coping mechanism because it has been positively related to healthy outcomes, such as social rehearsals [20], positive religious coping [4], death acceptance [21], selfesteem [3], and grit/resilience [22]. Conversely, the FAB has been disrupted by unhealthy outcomes, such as dysphoria [5], dispositional mood [23], trait anxiety [6], and narcissism [24]. The FAB has also been inhibited by alcohol consumption [25], marijuana consumption [26], death avoidance attitudes [27], disordered eating [28], and psychological distress variables [29].

Much like the research showing the FAB persisting across cultures [7], the literature has shown consistent FAB effects across event types. The FAB has been displayed across both control events and events involving alcohol [25], dreams [30], religion [4], significant events that involved deaths [22], social media [3], and videogames [9]. Although these studies generally showed FAB effects across target events (e.g., religious) and control events (e.g., non-religious), the FAB was low for certain target events, including social media events [3], videogame events [9], and religious events that did not involve spirituality [4].

Much like the FAB research showing a relation between emotions and religion [4], the literature on religion shows that it plays an important role in the well-being and perceived emotions of its adherents [31]. Specifically, religion is positively related to well-being [32, 33, 34], mental health and physical health [35], and it is negatively related to depression [36]. Based on the connection between religious variables and the FAB, the relation between religion and well-being, as well as the strong adherence to religious doctrines across cultures [31], the FAB was expected to differ across religious categories. This contention is based on the literature showing differences in emotions and measures of well-being across religious categories, which included Buddhists, Jews, Christians, Hindus, and Muslims.

The empirical research from psychological scientists on the emotions of adherents to religions has been underwhelming in terms of depth. The literature on Buddhists suggests that they show the greatest degree of positive emotions and the lowest degree of negative emotions compared to other religious affiliations, which may occur due to a high regard for contemplation and a penchant for seeking calmness and low arousal emotions [37]. Rözer and Kraaykamp [38] found that Buddhists experience greater subjective well-being than Christians and non-religious controls. In addition, Buddhists exhibit fewer depressive symptoms compared to non-religious controls [39] and they report lower levels of death anxiety compared to Muslims [40]. Maupin [41] discussed the fact that Zen Buddhism emphasizes the practice of detachment, where people separate themselves from the material world to focus on the present. As this practice is related to low anxiety [42], Buddhists should experience low levels of anxiety. The literature suggests that Buddhists should experience high levels of positive emotions and low levels of negative emotions.

Jewish tradition draws its inspiration from the Hebrew bible and the Talmud, and it emphasizes emotions across all manners of living, but the empirical literature on emotions and Judaism is mixed with a positive bias. Studies showed that Jews report high depression [43] and they experience religious struggles, which are related to mental health concerns and negative emotions [44], including depression, anxiety, and stress [45, 46, 47, 48]. In contrast, QuintanaOrts and Rey [49] demonstrated that Judaism regulates emotions, such as depression, and Ripley et al. [50] found that Judaism fosters positive mental health outcomes. Similarly, Fishbane [51] concluded that Jews embrace joy.

As Jews tend to be collective, they are more social and focused on the community than more individualistic religious groups, such as Christian Protestants [52] and they benefit in terms of emotional coping from community resources [53]. Judaism also emphasizes forgiveness, which is an emotion concerned with interpersonal relationships [54] and it is negatively related to anxiety [55]. In fact, research has shown that Jews report higher levels of forgiveness than Muslims [56, 57], and, along with Christians, they report lower levels of obsessive compulsions than Muslims [56]. As obsessive compulsions are connected to both anxiety [58] and depression [59], these results suggest that Jews and Christians experience low levels of negative emotions. The literature suggests that Jews tend to experience relatively strong positive emotions and weak negative emotions.

Certain Christians are likely to experience unpleasant emotions. For example, devout followers of Catholicism report greater feelings of guilt relative to non-religious controls [60,61,62,63]. Despite the negative connotations of guilt, it can lead to positive and cooperative coping mechanisms involving regard for other individuals [64]. In contrast, Christians seem to experience increased levels of positive emotions relative to practitioners of other religions. For example, Christians tend to embrace love and happiness [65] and they report greater subjective well-being than their 
non-Christian peers [66]. Christians also display lower death anxiety than Muslims and non-religious controls [40]. Interestingly, Christian children view God as more loving than Hindu or Muslim children [67]. As previously stated, Christians express more forgiveness [57] and lower levels of obsessive compulsions [56] than Muslims. Together, the findings suggest that Christians should experience strong positive emotions and weak negative emotions.

Hindu practitioners seem to experience negative emotions at a higher rate than followers of different religious affiliations. For example, one study showed that Hindus experienced more death anxiety than non-religious controls [40]. The distinctive, negative emotion that stands out for the Hindu religion seems to be shame [65]; one study showed that Hindus express significantly greater shame than Muslims [65]. However, Hinduism typically incorporates fasting practices, which are associated with mental clarity and emotional control [68], as well as mindfulness practices that help them and Buddhists deepen their connection with their environment [69], increase positive emotions, well-being, and mental health outcomes, as well as decrease psychological distress in the form of depression, anxiety, and stress $[70,71,72,73,74]$. Therefore, the Hindu ability to emotionally regulate, which is likely hampered by a susceptibility to experience negative emotions, may be reversed, and then enhanced by healthy practices interwoven within the religion.

Research suggests that adherents of Islam tend to experience negative emotions at a greater frequency compared to practitioners of other religions. Specifically, Muslims consistently report more sadness, and they seem to value anger and shame more than followers of other religions [75], except for Hindus [65]. Muslims are 4 times more likely to report symptoms of depression than controls [76], and they experience more death anxiety than Christians [77]. In addition, Muslim women report higher levels of death anxiety than Christians and non-Christian controls [40], and they experience stronger feelings of sexual guilt than women practicing other religions [78]. These negative emotions may occur because Islam practitioners tend to emphasize feelings of anger $[75,79,63]$ and Muslims report lower levels of forgiveness [80] and higher levels of obsessive compulsion [56] than Jews and Christians, which could lead to negative emotions.

In contrast to the research showing poor emotion regulation for Muslims, Kim-Prieto and Diener [65] found that followers of Islam seem to embrace positive emotions. In fact, Fischer et al. [81] found that Christians tend to look inward toward personal resources, such as personal spirituality with their God, to deal with depression, bereavement, physical illness, terrorism, and other life events, whereas Muslims tend to look toward the community and spiritual groups to deal with those same challenges. As interpersonal and community resources are superior coping mechanisms to intrapersonal resources [53], Muslims should regulate emotions across these challenges better than Christians. In support of this point, Haslam and Reicher [82] suggested that a sense of belonging and shared identity serve as a basis for affiliated members to collectively resist exposure to and the effects of negative emotions, such as anxiety and depression. The literature suggests that Muslims should experience a mix of negative and positive emotions, with a bias toward negative emotions.

In summary, the FAB is a healthy coping outcome that enhances pleasantness and reduces unpleasantness, it is positively related to healthy outcomes and negatively related to unhealthy outcomes, and it persists across many cultures and events. Importantly, the FAB is influenced by religion, such that spiritual events and events that are both religious and spiritual show robust $F A B$, but the FAB is not present for events that are religious, but not spiritual. The religion literature is congruent with these findings, showing that religion is important for well-being. Together, these results indicated that the FAB should differ across religious categories. Specifically, Buddhists should show strong FAB because they value and embrace moderate and calm emotions and Jews should show strong FAB because they embrace social connections and the emotions related to communal well-being.

Although Christians should show strong FAB because they embrace the pleasant emotions of love, happiness, arousal, and excitement, their preference for intense emotions suggests that pleasant affect may fade for them, which would result in moderate FAB. The prediction for Hindus is interesting but unclear because they express shame, which should negatively predict the FAB, but they also embrace forgiveness and mindfulness, which should positively predict the FAB. Although Muslims demonstrate superior coping mechanisms reliant on interpersonal and community resources, they tend to embrace and express more negative emotions than other religions, which predicts a low FAB for adherents of this religious affiliation. As the FAB literature shows that rehearsal mediates FAB effects [29], we expected rehearsal to mediate FAB effects across religious affiliations in the current study. 


\section{Methods}

\subsection{Participants}

Data were collected from repeated-measures designs with participant as the unit of analysis, but we analyzed the data with a between-groups design with event as the unit of analysis and we controlled for participant; this practice is normal in the FAB literature. The data came from FAB studies that examined the FAB across experimental events (e.g., alcohol) and control events (e.g., non-alcohol). Seven of these FAB studies were published, one of these FAB studies is currently under review, and two other studies are currently being written up for publication. The goal was to use the initial and final affect ratings and rehearsal ratings for events experienced by participants along with their religious affiliations for published and unpublished FAB studies that included these ratings. The study received approval from the Institutional Review Board at the university, which ensured that the procedure followed American Psychological Association (APA) guidelines [83], which included briefing, consent, and debriefing.

The data for the alcohol study came from four events (pleasant and unpleasant alcohol and non-alcohol events) provided by the 261 participants in the second study published by Gibbons et al. [25]. The data for the religion study came from the four events (pleasant and unpleasant religious and non-religious events) provided by the 130 participants in the study published by Gibbons et al. [4]. The data for the death study involved four events: pleasant regular events, unpleasant regular events, as well as pleasant significant events, and unpleasant significant events (death). The data were provided by the 247 participants in the study published by Gibbons et al. [21]. The data for the social media study came from the eight events ( 2 pleasant and 2 unpleasant social media events as well as 2 pleasant and 2 unpleasant non-social media events) provided by the 234 participants in the Gibbons et al. study [3]. The data for the human and pet death study came from the four events (two death events and two comparably emotionally intense, pleasant death events) provided by the 198 participants in the Gibbons et al. study [27]. The data from the videogame study came from the eight events ( 2 pleasant and 2 unpleasant videogame events as well as 2 pleasant and 2 unpleasant non-videogame events) provided by the 272 participants in the Gibbons and Bouldin study [9].

The data for the presidential election study came from four events (pleasant and unpleasant non-political events as well as the 2016 Presidential election [84] event and a comparably, but opposite, emotionally intense event) provided by 310 participants [85]. The data from the relationship study came from 12 events (3 pleasant and 3 unpleasant relationship events as well as 3 pleasant and 3 unpleasant non-relationship events) provided by 239 participants from the relationship study [86]. The data for the marijuana study came from eight events ( 2 pleasant and 2 unpleasant marijuana events as well as 2 pleasant and 2 unpleasant non-marijuana events) provided by 328 participants; the data from the marijuana study have not been published. The data from the videogame replication study came from 8 events ( 2 pleasant and 2 unpleasant videogame events as well as 2 pleasant and 2 unpleasant non-videogame events) provided by 140 participants; the data from the videogame study have not been published.

The study examined 15,108 events from 2,359 participants. The student sample ranged from 18 to 49 years old with most individuals under the age of 26 years, and comprised predominantly of White (roughly 80\%), Christian (roughly $80 \%$ ) women (roughly 70\%). The demographic variables for each published study are reported in those studies. As the relationship study is under review and not published yet, the sample of 239 participants was comprised of young adults (19.416 years), who were mainly Caucasian (75.5\%), Christian (77.5\%), heterosexual (94.4\%) women (77.9\%). As the marijuana study is unpublished, the participants were comprised of young adults (19.360 years) who were primarily Caucasian (74.57\%), Christian (74.6\%), heterosexual (87.8\%) women (80.60\%). As the second videogame study is unpublished, the participants were comprised of young adults (19.360 years) who were primarily Caucasian (82.7\%), Christian (72.9\%), heterosexual (89.4\%) women (60.0\% women). The total sample of 2,359 (15,108 events) participants was comprised of 11,508 Christian events, 196 Jewish events, 36 Muslim events, 60 Buddhist events, 80 Hindu events, 1,368 Agnostic events, 780 Atheist events, 512 Other events, and 568 No Label events.

\section{Materials, Procedures, Measures, Design, and Analyses}

The materials in each study included a questionnaire asking participants to provide brief event descriptions for pleasant and unpleasant events for each of the two different event types in each study (e.g., alcohol and non-alcohol, religious and non-religious, significant/death and non-significant, social media and non-social media, and pet/human death and non-death). Crossing initial event valence with event type created four kinds of events in each study. The participants were prompted to provide descriptions and ratings for each of the types of events in an order that was determined using a Latin square; participants were randomly assigned to the four different event orders. Participants were also asked to provide ratings of affect for each event at the time it initially occurred and at the time of the study on a scale ranging 
from -3 (very unpleasant) to +3 (very pleasant) with a 0 point (neutral). Although the political study was conducted within 3 months of the 2016 Presidential election, the other studies limited participants to events no older than 1 year. The time between initial event occurrence and test was only obtained in the election study [85].

Fading affect was the main measure for the study. For initially pleasant events, fading affect was calculated by subtracting the current affect from the original affect. For initially unpleasant events, fading affect was calculated by subtracting the original affect from the current affect. These calculations ensured that all measures of fading affect were positive across pleasant and unpleasant events, such that a large fading affect score indicated a large amount of fading, and a small fading affect score indicated little fading. Data were only used for initial event pleasantness ratings that matched the pleasantness of the rated events, such that the prompting of ratings for unpleasant events had to be rated by participants as initially unpleasant and the prompting of ratings for pleasant events had to be rated by participants as initially pleasant. The missing data points due to absent initial ratings or initial ratings that did not match the instructions as well as absent final ratings or final ratings that did not match instructions included 1517 (10.041\%) events out of 15,108 events, leaving 13,591 events.

A pure fading affect measure was calculated that eliminated flourishing affect events, which are events that became stronger in intensity [8], and flexible affect events, which are events that changed their affect from pleasant to unpleasant or vice versa [17]. We calculated and analyzed two measures to be complete and transparent. The 2,362 additional events that changed affect in an "unusual" way were removed from the remaining 13,591 (15.634\%) events, leaving 11,229 "pure" events. This loss of events was reasonable based on Gibbons and Rollins [84] who showed that $15.32 \%$ of their events across four experiments indicated "unusual" fading affect in the form of flourishing (increased) and flexible (changed to the other valence) affect. We also calculated event intensity for pleasant and unpleasant events by calculating the absolute value of the initial affect rating.

We examined regression-to-the-mean by calculating the correlation coefficient between the absolute value of the initial affect rating for pleasant and unpleasant events and each fading affect measure. Positive correlations would indicate regression to the mean. In addition, both the fading affect and pure fading affect measures were placed in a one-factor between-groups design with initial event affect (unpleasant and pleasant) as that factor. One-way between-groups ANCOVAs were conducted with fading affect and pure fading affect as the dependent variables, initial event affect as the independent variable, and the covariates included both initial event intensity (absolute value of the initial affect rating) and participant (a nominal variable). Although FAB research generally produces strong heterogeneity, violation of the parametric assumption of homogeneity for conducting ANOVA is not a problem if the sample sizes are relatively equal, defined by a ratio of largest to smallest sample sizes equal or less than 1.5 [87]. Overall and for each religious affiliation, the number of pleasant and unpleasant events were relatively equal and well within the 1.5 variance ratio limit.

\section{Results and Discussion}

Weak but significant regression-to-the mean was found for the relation of initially pleasant events and fading affect $(r(6803)=.232 p<.001)$ and it was found for the relations of initially unpleasant events and both fading affect ( $r(6784)$ $=.147, p<.001)$ and pure fading affect $(r(5197)=.187, p<.001)$. In addition, Table 1 showed that pleasant events were initially more intense than unpleasant events when fading affect could be calculated, except for Jews and Muslims. Similarly, Table 2 showed that pleasant events were initially more intense than unpleasant events when pure fading affect could be calculated, except for Muslims. The regression-to-the mean findings and the intensity differences in pleasant and unpleasant events indicate potential explanations for fading affect bias (FAB; greater fading of unpleasant affect than pleasant affect) effects that could challenge the internal validity of the FAB findings in the current study. However, the analyses examining the FAB eliminated these threats to internal validity by statistically controlling the initial intensity (absolute value) of initially pleasant and unpleasant events when examining the FAB across religious affiliations in the current study. 
Table 1 Initial Intensity for Pleasant and Unpleasant Events (SE) and Their F-value Comparison (When Fading Affect Could Be Calculated), as well as Fading Affect for Pleasant and Unpleasant Events (SE) and Their F-value Comparison and Effect Size Controlling for Initial Intensity.

\begin{tabular}{|l|l|l|l|l|l|l|l|}
\hline & $\begin{array}{l}\text { Pls. Intensity } \\
\text { Mean (SE) }\end{array}$ & $\begin{array}{l}\text { Unpls. } \\
\text { Intensity } \\
\text { Mean (SE) }\end{array}$ & $\begin{array}{l}\text { Intensity F- } \\
\text { value (p) }\end{array}$ & $\begin{array}{l}\text { Pls. Change } \\
\text { (SE) }\end{array}$ & $\begin{array}{l}\text { Unpls. } \\
\text { Change (SE) }\end{array}$ & $\begin{array}{l}\text { FAB F-value } \\
(\boldsymbol{p})\end{array}$ & $\begin{array}{l}\text { Partial } \\
\boldsymbol{E}^{2}\end{array}$ \\
\hline Jewish & $2.473(0.078)$ & $2.283(0.079)$ & $2.906(.090)$ & $0.450(0.153)$ & $2.091(0.154)$ & $57.090^{* * *}$ & .247 \\
\hline Buddhist & $2.831(0.104)$ & $2.381(0.106)$ & $9.128(.004)$ & $0.297(0.247)$ & $1.589(0.251)$ & $13.475^{* * *}$ & .194 \\
\hline Hindu & $2.770(0.098)$ & $2.435(0.098)$ & $5.875(.018)$ & $0.462(0.230)$ & $1.641(0.230)$ & $13.159(.001)$ & .149 \\
\hline Christian & $2.590(0.010)$ & $2.377(0.010)$ & $230.206^{* * *}$ & $0.512(0.019)$ & $1.647(0.019)$ & $1746.752^{* * *}$ & .145 \\
\hline Atheist & $2.507(0.039)$ & $2.308(0.040)$ & $12.602^{* * *}$ & $0.469(0.071)$ & $1.517(0.072)$ & $107.908^{* * *}$ & .129 \\
\hline Other & $2.521(0.048)$ & $2.316(0.048)$ & $8.982(.003)$ & $0.664(0.092)$ & $1.687(0.092)$ & $61.776^{* * *}$ & .113 \\
\hline Agnostic & $2.482(0.029)$ & $2.331(0.029)$ & $13.584^{* * *}$ & $0.587(0.054)$ & $1.419(0.054)$ & $120.100^{* * *}$ & .088 \\
\hline No Label & $2.482(0.049)$ & $2.316(0.048)$ & $7.361(.007)$ & $0.452(0.095)$ & $1.334(0.095)$ & $42.958^{* * *}$ & .086 \\
\hline Islam & $2.600(0.183)$ & $2.385(0.197)$ & $0.646(.430)$ & $0.952(0.387)$ & $0.806(0.418)$ & $0.065(.800)$ & .003 \\
\hline
\end{tabular}

The results for fading affect are presented in Table 1 and the results for pure fading affect (no flourishing affect or flexible affect) are presented in Table 2. Both tables present the results in descending order based on effect size and they generally tell a similar story with some minor differences. Specifically, the FAB was reliable for fading affect and pure fading affect across every religious affiliation except for Islam; the small sample of adherents to Islam in the current study likely explained the lack of FAB for this religious affiliation. For the fading affect measure, the strongest to weakest FAB effects were Jews, Buddhists, Hindus, Christians, Atheists, Others, Agnostics, No Label, and Islam/Muslims. For the pure fading affect measure, the strongest to weakest effects were Buddhists, Jews, Hindus, Islam/Muslims, Christians, Atheists, Other, Agnostics, and No Label. Together, the results suggest that the FAB effects range from strongest to weakest in the following order: Jews and Buddhists, Hindus, Christians, Atheists and Muslims, Other, Agnostic, and No Label. These findings generally matched our hypothesis based on the literature. However, the results did not exactly match our hypothesis that Buddhists would show the strongest FAB followed by Jews, Christians, Hindus, and Muslims. The findings for the Atheists, Agnostics, and participants who did not adopt a well-known religious affiliation were a surprise due to a scarcity of literature examining religious affiliations and healthy/unhealthy emotions and outcomes.

Table 2 Initial Intensity for Pleasant and Unpleasant Events (SE) and Their F-value Comparison (When Pure Fading Affect Could Be Calculated), as well as Pure Fading Affect for Pleasant and Unpleasant Events (SE) and Their F-value Comparison and Effect Size Controlling for Initial Intensity.

\begin{tabular}{|l|l|l|l|l|l|l|l|}
\hline & $\begin{array}{l}\text { Pls. } \\
\text { Intensity } \\
\text { Mean (SE) }\end{array}$ & $\begin{array}{l}\text { Unpls. } \\
\text { Intensity } \\
\text { Mean (SE) }\end{array}$ & $\begin{array}{l}\text { Intensity F- } \\
\text { value (p) }\end{array}$ & $\begin{array}{l}\text { Pls. Change } \\
\text { (SE) }\end{array}$ & $\begin{array}{l}\text { Unpls. } \\
\text { Change (SE) }\end{array}$ & $\begin{array}{l}\text { FAB F-value } \\
(\boldsymbol{p})\end{array}$ & $\begin{array}{l}\text { Partial } \\
\boldsymbol{E}^{2}\end{array}$ \\
\hline Buddhist & $2.890(0.084)$ & $2.613(0.093)$ & $4.861(.032)$ & $0.215(0.159)$ & $1.173(0.175)$ & $16.344^{* * *}$ & .254 \\
\hline Jewish & $2.639(0.076)$ & $2.394(0.087)$ & $4.474(.036)$ & $0.580(0.095)$ & $1.350(0.109)$ & $28.292^{* * *}$ & .179 \\
\hline Hindu & $2.811(0.095)$ & $2.500(0.109)$ & $4.608(.036)$ & $0.438(0.121)$ & $1.064(0.140)$ & $11.446(.001)$ & .156 \\
\hline Islam & $2.612(0.199)$ & $2.514(0.207)$ & $0.119(.734)$ & $0.443(0.259)$ & $1.062(0.270)$ & $2.732(.114)$ & .120 \\
\hline Christian & $2.704(0.009)$ & $2.498(0.010)$ & $225.152^{* * *}$ & $0.557(0.014)$ & $1.201(0.015)$ & $1021.981^{* * *}$ & .107 \\
\hline Atheist & $2.645(0.037)$ & $2.475(0.041)$ & $9.374(.002)$ & $0.581(0.051)$ & $1.192(0.056)$ & $64.771^{* * *}$ & .099 \\
\hline Other & $2.640(0.047)$ & $2.461(0.052)$ & $6.600(.011)$ & $0.651(0.064)$ & $1.242(0.070)$ & $38.871^{* * *}$ & .092 \\
\hline Agnostic & $2.604(0.028)$ & $2.459(0.029)$ & $12.849^{* * *}$ & $0.632(0.040)$ & $1.152(0.041)$ & $81.670^{* * *}$ & .072 \\
\hline No Label & $2.594(0.048)$ & $2.387(0.050)$ & $8.943(.003)$ & $0.497(0.064)$ & $0.990(0.066)$ & $28.898^{* * *}$ & .071 \\
\hline
\end{tabular}


The current study is important because it is the first study to examine the FAB across religious affiliations. The results of the current study show strong FAB effects across every religious affiliation with a sufficient sample, even though the strength of those effects differed across those affiliations. Much like the research demonstrating the FAB across cultures [7], the FAB seems to be present across every religious affiliation. These results replicate and extend past work on the FAB that uses Taylor's [19] mobilization-minimization hypothesis to explain FAB effects. Specifically, once someone encounters an emotional experience, their body activates its resources (e.g., the release of endorphins and serotonin, as well as the decrease of epinephrine and cortisol) to diminish unpleasant experiences and heighten pleasant ones. These findings also fit well with work on the FAB suggesting that the phenomenon is an outcome of self-enhancement strategies that make life pleasant by increasing the pleasantness of past events [88, 18]. Therefore, the emotionregulating and self-enhancing effect that is the FAB pervades, and yet differs, across the different religious affiliations.

When the patterns from the two tables are combined, they suggest a strong FAB group, a less strong FAB group, and a weak FAB group. Jews and Buddhists consistently showed the strongest FAB across the two measures, whereas Hindus and Christians showed the next strongest FAB effects. The groups showing the weakest FAB included participants who provided No Label and identified as Agnostic followed by participants who identified as Other. Atheists and Muslims were found in the middle. Although Muslims showed a low FAB due to low sample size, Other, No Label, and Agnostic participants showed low FAB effects even though they were represented by large samples. These three groups do not subscribe to well-known religious affiliations that advocate specific beliefs or the lack of belief. In contrast, individuals in these three groups did not commit to a well-known religious affiliation with a particular religious viewpoint/belief, and their absence of commitment, allegiance, and connectedness with individuals sharing their thoughts and beliefs may explain their poor emotion regulation in the form of weak FAB.

The results suggest that individuals who have pledged their allegiance to a particular religious indoctrination, belief, and affiliation benefit from medium to large FAB effects. The importance of a religious affiliation that adheres to a particular belief for FAB effects may be due to the official connection to an established group or an opportunity to share thoughts and beliefs with likeminded individuals. The opportunity to share one's events with other individuals is important in the FAB literature, as the FAB is positively related to increased social sharing [89], especially when sharing with attentive listeners [20]. Future research should determine the deeper underpinnings and explanatory mechanisms for these findings.

Although the studies used to produce the data in the current study were not designed to examine many different types of mediators for its FAB effects across religious affiliations, they all included rehearsal (thinking or talking) ratings, which have been explanatory mechanisms for past FAB effects $[3,29,20,88,89]$. Therefore, we examined the importance of rehearsal ratings for the FAB effects across religious affiliations in the current study by statistically removing them as a covariate from the FAB effects for each religious affiliation in the current study. Even though the resulting FAB effects were weaker after the statistical removal of rehearsals, the reductions were small, and the FAB effects remained both significant and strong, except for Muslims, after the statistical adjustment.

As rehearsals could only account for, at best, a small part of the FAB effects across religious affiliations found in the current study, future research should examine alternative explanations for FAB effects across religious affiliations. For example, future research could examine biological mechanisms, such as serotonin, dopamine, endorphins, norepinephrine, cortisol, and brain activity (e.g., low activity in the amygdala and high activity in the hypothalamus or pre-frontal cortex). Future research could also examine social activity, group cohesion, religious belief, and religious practice (called religiosity) as mediators for FAB effects across religious affiliations. In fact, Gibbons et al. [4] found that spirituality was much more important for $\mathrm{FAB}$ effects than religiosity. Therefore, future research examining the $\mathrm{FAB}$ across religious affiliation should account for spirituality as well. The identification of these mechanisms could point to attributes that could hinder the FAB and target individuals for therapeutic assistance and it could determine techniques that could aid clients or therapists in therapy for maladaptive emotions and psychological distress.

The major limitation in the current study pertained to the low sample of Islamic followers. Future research can correct this limitation by gathering a sufficiently large sample (e.g., at least 40 pleasant and 40 unpleasant events) of Muslims and examine the FAB. Unpleasant affect will likely fade significantly more than pleasant affect, demonstrating the FAB, but the effect sizes for Islam in the current study cast doubt on the strength of the FAB effects to be found for this religious affiliation. The most probable outcome is that the FAB for Islamic followers will likely demonstrate low to medium effect sizes. Although the FAB was statistically reliable and the effect sizes were large for Jews, Buddhists, and Hindus, the number of individuals subscribing to each of these religious affiliations was not large in the current study. Therefore, future research should increase the sample of Jews, Buddhists, Hindus, and Muslims to represent them better than they were represented in the current study. Based on the results of the current study, robust FAB effects with 
strong effect sizes should be found for Jews, Hindus, and Buddhists, whereas the FAB may be less strong, but still significant, for Muslims.

Except for Muslims, the significant FAB effects for every religious affiliation demonstrated internal validity for all these religious affiliations. Although larger samples of Muslims, Buddhists, Hindus, and Jews are needed in future research to demonstrate generalizability via replication, the large sample sizes of some religious affiliations in the current study, defined by samples exceeding 50 participants, begin the process of generalizability via representation. Future research should extend the procedure used in the current study to various regions in the United States as well as a diverse assortment of different countries around the world.

The retrospective methodology used in the current study often receives criticism for its lack of validity. As human lives are always described retrospectively in conversations with friends and family at gatherings, which include the birth of children, firsts (e.g., first words or teeth), graduations, weddings, anniversaries, retirements, and funerals, the method certainly curries external validity in the form of ecological validity. However, one may still worry that retrospective procedures are inaccurate and potentially uncover effects that are not real by overestimating the existence or importance of effects. The FAB literature has put this worry to rest, showing that retrospective methodology provides FAB effects that match or underestimate the FAB [17]. Specifically, Ritchie et al. [17] found that retrospective methods underestimated the initial affect ratings for unpleasant events gathered in a diary study, which means that retrospective methodologies underestimate the fading affect for unpleasant events, and they underestimate the FAB. Therefore, the FAB effects found in studies using retrospective methodologies are real, but they may not be accurate, as they are likely much smaller in the current study than they would be if they were examined with diary methodology.

Another criticism that could be levied against the current study is its use of single-item affect scales. Although singleitem measures display some disadvantages compared to multi-item scales, single-item measures are efficient, they can yield comparable psychometric qualities to multi-item instruments [90], and they are commonly used in emotion research because of their economy and interpretability $[91,92,93]$. In addition, the method of measurement used in our study is a standard approach in the FAB literature $[25,4,17,88,89,1]$. If we adopted a different measurement system than the one commonly used in FAB research, our results would be questionable. Moreover, the use of multiitem scales, like the 10-item Affect Balance Scale, would be burdensome and cumbersome for participants rating 8 to 12 events. The repetitiveness of completing these scales with many events could also cause fatigue and lead to response sets, which would harm the internal validity of the results.

\section{Conclusion}

In summary, the current study is the first one to examine the FAB across religious affiliations and it showed that the FAB was significant across all religious categories, except for Islam due to a low sample of participants. Importantly, FAB differed across religious affiliations, being especially strong for Jews and Buddhists followed by Hindus and Christians and they were particularly weak for the small sample of Muslims and for participants who did not select a well-known religious affiliation committed to a particular belief or lack of belief. Therefore, future research should replicate the current study, increase the sample size of Jews, Buddhists, Hindus, and Muslims to better represent them, and examine mediators for the FAB effects that should include, but not be limited to, biological substrates, cognitions (i.e., purposefully thinking about events), cognitive mechanisms (e.g., working memory), as well as event sharing and group connectivity to explain these effects. The identification of these mechanisms for emotion regulation in the form of the FAB should improve scientists' understanding of emotional control and it could help therapists develop strategies to reduce the psychological distress experienced by their clients.

\section{Compliance with ethical standards}

\section{Acknowledgments}

We would like to thank the many research assistants who have worked in the Basic and Applied Memory and Cognition Laboratory at Christopher Newport University over the past 8 years (roughly 80 undergraduate students) who helped run the studies and collect the data that were analyzed and presented in the current study.

\section{Disclosure of conflict of interest}

The authors of this study have no conflict of interest. 


\section{Statement of informed consent}

Informed consent was obtained from all individual participants included in the study.

\section{References}

[1] Walker WR, Vogl RJ, Thompson CP. Autobiographical memory: Unpleasantness fades faster than pleasantness over time. Applied Cognitive Psychology. 1997; 11: 399-413.

[2] Walker WR, Skowronski JJ, Thompson CP. Life is pleasant - and memory helps to keep it that way! Review of General Psychology. 2003; 7: 203-210.

[3] Gibbons JA, Horowitz KA, Dunlap SM. Does the fading affect bias show healthy coping at the general and individual levels in the context of social media? Paper submitted to Consciousness and Cognition. 2017.

[4] Gibbons JA, Hartzler JK, Hartzler AW, Lee SA, Walker WR. The fading affect bias shows healthy coping at the general level, but not the specific level for religious and non-religious events. Consciousness and Cognition. 2015; 36: 265-276.

[5] Walker WR, Skowronski JJ, Gibbons JA, Vogl RJ, Thompson CP. On the emotions that accompany autobiographical memories: Dysphoria disrupts the fading affect bias. Cognition \& Emotion. 2003; 17: 703-723.

[6] Walker WR, Yancu CN, Skowronski JJ. Trait anxiety reduces affective fading for both positive and negative autobiographical memories. Advances in Cognitive Psychology. 2014; 10: 81-89.

[7] Ritchie TD, Batteson TJ, Bohn A, Crawford MT, Ferguson GV, Schrauf RW, Walker WR. A pancultural perspective on the fading affect bias in autobiographical memory. Memory. 2014a; 23: 278-290.

[8] Walker WR, Skowronski JJ. The fading affect bias: But what the hell is it for? Applied Cognitive Psychology. 2009; 23: 1122-1136.

[9] Gibbons JA, Bouldin B. Videogame play and events are related to unhealthy emotion regulation in the form of low fading affect bias in autobiographical memory. Consciousness and cognition. 2019 Sep 1; 74: 102778.

[10] Jersild A. Memory for the pleasant as compared with the unpleasant. Journal of Experimental Psychology. 1931; 14: 284-288.

[11] Meltzer H. Individual differences in forgetting pleasant and unpleasant experiences. Journal of Educational Psychology. 1930; 21: 399-409.

[12] Meltzer H. Sex differences in forgetting pleasant and unpleasant experiences. Journal of Abnormal and Social Psychology. 1931; 25: 450-464.

[13] Watters R, Leeper R. The relation of affective tone to the retention of experiences in everyday life. Journal of Experimental Psychology. 1936; 19: 203-215.

[14] Cason H. The learning and retention of pleasant and unpleasant activities. Archives of Psychology. 1932; 134: 96.

[15] Holmes D. Differential change in affective intensity and the forgetting of unpleasant personal experiences. Journal of Personality and Social Psychology. 1970; 15: 234-239.

[16] Gibbons JA, Lee SA, Walker R. The fading affect bias begins within 12 hours and persists for 3 months. Applied Cognitive Psychology. 2011; 25: 663-672.

[17] Ritchie TD, Skowronski JJ, Harnett J, Wells B, Walker WR. The fading affect bias in the context of emotion activation level, mood, and personal theories of emotion change. Memory. 2009; 17: 428-444.

[18] Sedikides C, Alicke MD. The five pillars of self-enhancement and self-protection. In R. M. Ryan (Ed.). Oxford handbook of motivation. 2018.

[19] Taylor SE. Asymmetrical effects of positive and negative affect: The mobilization-minimization hypothesis. Psychological Bulletin. 1991; 110: 67-85.

[20] Muir K, Brown C, Madill A. The fading affect bias: Effects of social disclosure to an interactive versus nonresponsive listener. Memory. 2014; 23: 829-847.

[21] Gibbons JA, Fehr AMA, Brantley JC, Wilson KJ, Lee SA, Walker WR. Testing the fading affect bias for healthy coping in the context of death. Death Studies. 2016. 
[22] Zengel B, Lee EM, Walker WR, Skowronski JJ. Romantic relationships and fading of affect for memories of the shared past. Applied Cognitive Psychology. 2019 Sep; 33(5):861-72.

[23] Ritchie T, Skowronski JJ, Hartnett J, Wells B, Walker WR. The fading affect bias in the context of emotion activation level, mood, and personal theories of emotion change. Memory. 2009 Apr 1; 17(4):428-44.

[24] Ritchie TD, Walker WR, Marsh S, Hart C, Skowronski JJ. Narcissism distorts the fading affect bias in autobiographical memory. Applied Cognitive Psychology. 2014; 29: 104-114.

[25] Gibbons JA, Toscano A, Kofron S, Rothwell C, Lee SA, Ritchie TD, Walker WR. The fading affect bias across alcohol consumption frequency for alcohol-related and non-alcohol-related events. Consciousness and cognition. 2013 Dec 1;22(4):1340-51.

[26] Pillersdorf D, Scoboria A. Cannabis-associated impairments in the fading affect bias and autobiographical memory specificity. Consciousness and Cognition. 2019; 74.

[27] Gibbons JA, Lee SA, Fehr A, Wilson KJ, Marshall TR. Grief and avoidant death attitudes combine to predict the fading affect bias. International journal of environmental research and public health. 2018 Aug; 15(8): 1736

[28] Ritchie TD, Kitsch KS, Dromey M, Skowronski JJ. Individuals who report eating disorder symptoms also exhibit a disrupted fading affect bias in autobiographical memory. Memory. 2019; 27: 239-249.

[29] Gibbons JA, Lee SA. Rehearsal partially mediates the negative relations of the fading affect bias with depression, anxiety, and stress. Applied Cognitive Psychology. 2019 Jul; 33(4): 693-701.

[30] Ritchie TD, Skowronski JJ. Perceived change in the affect associated with dreams: The fading affect bias and its moderators. Dreaming. 2008; 18: 27-43.

[31] Green M, Elliott M. Religion, health, and psychological well-being. Journal of Religion and Health. 2010; 49: 149163.

[32] Elliott M, David Hayward R. Religion and well-being in a church without a creed. Mental Health, Religion \& Culture. 2007 Mar 1; 10(2): 109-26.

[33] Ellison CG. Religious involvement and subjective well-being. Journal of Health and Social Behavior. 1991; 32: 80-99.

[34] Steger MF, Frazier P. Meaning in life: One link in the chain from religiousness to well-being. Journal of Counseling Psychology. 2005; 52: 574-582.

[35] Abdel-Khalek AM, Naceur F. Religiosity and its association with positive and negative emotions among college students from Algeria. Mental Health, Religion \& Culture. 2007; 10: 159-170.

[36] Schnittker J. When is faith enough? The effects of religious involvement on depression. Journal for the scientific study of religion. $2001 ; 40: 393-411$

[37] Emmons RA. The psychology of ultimate concerns: Motivation and spirituality in personality. Guilford Press. 1999.

[38] Rözer J, Kraaykamp G. Income inequality and subjective well-being: A cross-national study on the conditional effects of individual and national characteristics. Social indicators research. 2013 Sep 1; 113(3): 1009-23.

[39] O'Connor LE, Berry JW, Stiver DJ, Rangan RK. Depression, guilt, and Tibetan Buddhism. Psychology. 2012; 3: 805.

[40] Ellis L, Wahab EA, Ratnasingan M. Religiosity and fear of death: A three-nation comparison. Mental Health, Religion, \& Culture. 2013; 16: 179-199.

[41] Maupin EW. (1962). Zen Buddhism: A psychological review. Journal of Consulting Psychology. $1962 ; 26: 362$.

[42] Kalisch R, Wiech K, Critchley HD, Seymour B, O'doherty JP, Oakley DA, Dolan RJ. Anxiety reduction through detachment: subjective, physiological, and neural effects. Journal of Cognitive Neuroscience. 2005; 17: 874-883.

[43] Koenig HG. Religion as cognitive schema. The International Journal for the Psychology of Religion. 1995; 5: 3137.

[44] Exline JJ. Religious and spiritual struggles. APA Handbook of Psychology, Religion, and Spirituality. 2013; 1: 459-475. 
[45] Ano GG, Vasconcelles EB. Religious coping and psychological adjustment to stress: A meta-analysis. Journal of Clinical Psychology. 2005; 61: 461-480.

[46] Harris JI, Erbes CR, Engdahl BE, Ogden H, Olson RH, Winskowski AMM, Mataas S. Religious distress and coping with stressful life events: A longitudinal study. Journal of Clinical Psychology. 2012; 68: 1276-1286.

[47] Wilt JA, Grubbs JB, Exline JJ, Pargament KI. Personality, religious and spiritual struggles, and well-being. Psychology of Religion and Spirituality. 2016.

[48] Wilt JA, Grubbs JB, Pargament KI, Exline JJ. Religious and spiritual struggles, past and present: Relations to the big five and well-being. The International Journal for the Psychology of Religion. 2016.

[49] Quintana-Orts C, Rey L. Forgiveness, depression, and suicidal behavior in adolescents: Gender differences in his relationship. The Journal of Genetic Psychology. 2018; 179: 85-89.

[50] Ripley J, Worthington E, Garthe R, Davis D, Hook J, Reid C, Akpalu B. Trait forgiveness and dyadic adjustment predict postnatal depression. Journal of Child and Family Studies. 2018; 27: 2185-2192.

[51] Fishbane MA. The exegetical imagination: On Jewish thought and theology. Harvard University Press. 1998.

[52] Cohen AB, Hill PC. Religion as culture: religious individualism and collectivism among American Catholics, Jews, and Protestants. Journal of Personality. 2007; 75(4): 709-742.

[53] Gross JJ, Jazaieri H. Emotion, emotion regulation, and psychopathology: an affective science perspective. Clinical Psychological Science. 2014; 2: 387-401.

[54] Dratch RM. Forgiving the unforgivable? Jewish insights into repentance and forgiveness. Journal of Religion \& Abuse. 2003; 4: 7-24.

[55] Lee HM, Chen MT. Assessment of potential relationship between forgiveness and anxiety. Bulletin of Educational Psychology. 2009.

[56] Dèttore D, Berardi D, Pozza A. Religious affiliation and obsessive cognitions and symptoms: A comparison between Jews, Christians, and Muslims in non-clinical groups in Italy. Psychology of Religion and Spirituality. 2017; 9: 348.

[57] Fox A, Thomas T. Impact of religious affiliation and religiosity on forgiveness. Australian Psychologist. 2008; 43: 175-185.

[58] Tükel R, Polat A, Özdemir Ö, Aksüt D, Türksoy N. Comorbid conditions in obsessive-compulsive disorder. Comprehensive Psychiatry. 2002; 43: 204-209.

[59] Overbeek T, Schruers K, Vermetten E, Griez E. Comorbidity of obsessive-compulsive disorder and depression: Prevalence, symptom severity, and treatment effect. The Journal of Clinical Psychiatry. 2002; 63: 1106-1112.

[60] Albertsen EJ, O'Connor LE, Berry JW. Religion and interpersonal guilt: Variations across ethnicity and spirituality. Mental Health, Religion \& Culture. 2006; 9: 67-84.

[61] Stearns PN, Lewis J, editors. An emotional history of the United States. NYU Press; 1998.

[62] Walinga P, Corveleyn J, van Saane J. Guilt and religion: The influence of Othodox Protestant and Orthodox Catholic conceptions of guilt on guilt. Archive for the Psychology of Religion. 2005; 27: 113-135.

[63] Watts F. Emotion regulation and religion. Handbook of emotion regulation. 2007; 504-520.

[64] Behrendt H, Ben-Ari R. The positive side of negative emotion: The role of guilt and shame in coping with interpersonal conflict. Journal of Conflict Resolution. 2012; 56: 1116-1138

[65] Kim-Prieto C, Diener E. Religion as a source of variation in the experience of positive and negative emotions. The Journal of Positive Psychology. 2009; 4: 447-460.

[66] Bowman NA, Small JL. Exploring a hidden form of minority status: College students' religious affiliation and well-being. Journal of College Student Development. 2012; 53: 491-509.

[67] Takriti RA, Barrett M, Buchanan-Barrow E. Children's understanding of religion: Interviews with Arab-Muslim, Asian-Muslim, Christian and Hindu children aged 5-11 years. Mental Health, Religion \& Culture. 2006; 9: 2942.

[68] Rajendran NS. Science of fasting: Aspects from Hinduism perspective. Fasting and Sustainable Health Conference. 2010. 
[69] Sturm D, Presbury J, Etcherling L. The elements: a model of mindful supervision. Journal of Creativity in Mental Health. 2012; 7: 222-232.

[70] Brown KW, Ryan RM. The benefits of being present: mindfulness and its role in psychological well-being. Journal of Personality and Social Psychology. 2003; 84: 822-848.

[71] Jimenez SS, Niles BL, Park CL. A mindfulness model of affect regulation and depressive symptoms: positive emotions, mood regulation expectancies, and self-acceptance as regulatory mechanisms. Personality and Individual Differences. 2010; 49: 645-650.

[72] Kabat-Zinn J. Full Catastrophe Living: The Program of the Stress Reduction Clinic at the University of Massachusetts Medical Center. New York, NY: Delta. 1990.

[73] Segal ZV, Teasdale JD, Williams JM, Gemar MC. The mindfulness-based cognitive therapy adherence scale: interrater reliability, adherence to protocol and treatment distinctiveness. Clinical Psychology \& Psychotherapy. 2002; 9: 131-138.

[74] Soysa CK, Wilcomb CJ. Mindfulness, self-compassion, self-efficacy, and gender as predictors of depression, anxiety, stress, and well-being. Mindfulness. 2015; 6: 217-226.

[75] Abu-Lughod L. Veiled sentiments: Honor and poetry in a Bedouin society. University of California Press. 2016.

[76] Williams R, Hunt K. Psychological distress among British South Asians: the contribution of stressful situations and subcultural differences in the West of Scotland Twenty-07 Study. Psychological medicine. 1997 Sep;27(5):1173-81.

[77] McAdie T, Morris GJ. Are personality, well-being and death anxiety related to religious affiliation? Mental Health, Religion, and Culture. 2009; 12: 115-120.

[78] Abdolsalehi-Najafi E, Beckman LJ. Sex guilt and life satisfaction in Iranian-American women. Archives of Sexual Behavior. 2013; 42: 1063-1071.

[79] Appadurai A. Gratitude as a social mode in South India. Ethos. 1985; 13: 236-245.

[80] Mullet E, Azar F. Apologies, repentance, and forgiveness: A Muslim-Christian comparison. The International Journal for the Psychology of Religion. 2009; 19(4): 275-280.

[81] Fischer P, Ai AL, Aydin N, Frey D, Haslam SA. The relationship between religious identity and preferred coping strategies: An examination of the relative importance of interpersonal and intrapersonal coping in Muslim and Christian faiths. Review of General Psychology. 2010 Dec;14(4):365-81.

[82] Haslam SA, Reicher SD. Stressing the group: Social identity and the unfolding dynamics of stress. Journal of Applied Psychology. 2006; 91: 1037-1052.

[83] Publication Manual of the American Psychological Association (6th ed., pp. 20). Washington, DC: American Psychological Association. 2010.

[84] Gibbons JA, Rollins L. Assessing the Initial Pleasantness for Fading Affect, Fixed Affect, Flourishing Affect, and Flexible Affect Events. Applied Cognitive Psychology. 2016 Nov;30(6):1100-5.

[85] Gibbons JA, Dunlap S, LeRoy S, Thomas T. Conservatism positively predicted fading affect bias in the 2016 US presidential election at low, but not high, levels of negative affect. Applied Cognitive Psychology. 2020; 25(4).

[86] Gibbons JA, Friedmann E, Harris K. Examining the relation of the fading affect bias to marijuana consumption across marijuana and non-marijuana events. Manuscript in preparation. 2021.

[87] Statistics Solutions. The assumption of homogeneity of variance. 2018.

[88] Ritchie TD, Skowronski JJ, Wood S, Walker WR, Vogl RJ, Gibbons JA. Event self-importance, event rehearsal, and the fading affect bias in autobiographical memory. Self and Identity. 2006; 5: 172-195.

[89] Skowronski JJ, Walker WR. How describing autobiographical events can affect autobiographical memories. Social Cognition. 2004 Oct 1;22(5: Special issue):555-90.

[90] Bergkvist L, Rossiter JR. The predictive validity of multiple-item versus single item measures of the same constructs. Journal of Marketing Research. 2007; 44(2): 175-184.

[91] Cheung F, Lucas RE. Assessing the validity of single-item life satisfaction measures: Results from three large samples. Quality of Life Research. 2014; 23(10): 2809-2818. 
World Journal of Advanced Research and Reviews, 2021, 09(03), 350-362

[92] Lee SA. Coronavirus Anxiety Scale: A brief mental health screener for COVID-19 related anxiety. Death Studies. 2020; 44(7): 393-401.

[93] Robins RW, Hendin HM, Trzesniewski KH. Measuring Global Self-Esteem: Construct validation of a single-item measure and the Rosenberg Self-Esteem Scale. Personality and Social Psychology Bulletin. 2001; 27: 151-161. 